\title{
A crucial epithelial to mesenchymal transition regulator, Sox4/Ezh2 axis is closely related to the clinical outcome in pancreatic cancer patients
}

\author{
SHINICHIRO HASEGAWA ${ }^{1,2}$, HIROAKI NAGANO ${ }^{1}$, MASAMITSU KONNO ${ }^{2}$, HIDETOSHI EGUCHI $^{1}$, \\ AKIRA TOMOKUNI ${ }^{1}$, YOSHITO TOMIMARU ${ }^{1}$, TADAFUMI ASAOKA ${ }^{1}$, HIROSHI WADA ${ }^{1}$, \\ NAOKI HAMA $^{1}$, KOICHI KAWAMOTO ${ }^{1}$, SHIGERU MARUBASHI ${ }^{1}$, NAOHIRO NISHIDA ${ }^{2}$, \\ JUN KOSEKI $^{3}$, MASAKI MORI ${ }^{1}$, YUICHIRO DOKI ${ }^{1}$ and HIDESHI ISHII ${ }^{2,3}$ \\ Departments of ${ }^{1}$ Gastroenterological Surgery, ${ }^{2}$ Frontier Science for Cancer and Chemotherapy, \\ ${ }^{3}$ Cancer Profiling Discovery, Osaka University Graduate School of Medicine, Suita, Osaka 565-0871, Japan
}

Received July 23, 2015; Accepted September 7, 2015

DOI: 10.3892/ijo.2015.3258

\begin{abstract}
Pancreatic cancer has a poor prognosis because of its high invasiveness and recurrence, and these properties closely link to the phenomenon of epithelial-mesenchymal transition (EMT). Recently, it has been reported that Sox4 is indispensable for EMT in vitro and in vivo and regulates various master regulators of EMT including Zeb, Twist and Snail. Moreover, Sox4 induces the transcription of Ezh2 which is the histone methyltransferase, and reprograms the cancer epigenome to promote EMT and metastasis. Therefore, the present study evaluated the importance of Sox4, Ezh2 and miR-335, which regulate Sox 4 expression epigenetically, in clinical samples with pancreatic cancer. This retrospective analysis included data from 36 consecutive patients who underwent complete surgical resection for pancreatic cancer and did not undergo any preoperative therapies. We assessed the clinical significance of Sox4/Ezh2 axis and miR-335 expression, using immunohistochemistry and qRT-PCR with laser captured microdissection (LCM). The Sox 4 positive patients had significantly worse prognosis as for disease-free survival (DFS) $(\mathrm{P}=0.0154)$ and the Ezh2-positive patients had significantly worse prognosis as for overall survival (OS) $(\mathrm{P}=0.0347)$. The miR-335 expression was inversely correlated
\end{abstract}

Correspondence to: Professor Hideshi Ishii, Department of Cancer Profiling Discovery, Osaka University Graduate School of Medicine, 2-2, Yamadaoka, Suita, Osaka 565-0871, Japan

E-mail: hishii@gesurg.med.osaka-u.ac.jp

Professor Masaki Mori, Department of Gastroenterological Surgery, Osaka University Graduate School of Medicine, 2-2, Yamadaoka, Suita, Osaka 565-0871, Japan

E-mail: mmori@gesurg.med.osaka-u.ac.jp

Key words: Sox4, Ezh2, miR-335, pancreatic cancer, prognosis, biomarker, immunohistochemistry, laser captured microdissection with Sox4 expression in the identical clinical specimens, but it was not related to the prognosis. Sox $4 /$ Ezh2 axis was closely associated with the prognosis in pancreatic cancer patients.

\section{Introduction}

Pancreatic cancer has the worst prognosis of all the major types of cancers and it is the fourth leading cause of cancer death (1). The survival ratio of 5 years in pancreatic cancer approximates $5 \%$ (2). This miserable lethality is caused by the difficulty of diagnosis at an early operable stage and the aggressiveness of the cancer cells $(3,4)$. Even if it is diagnosed at an early stage and resected curatively, there is still a high incidence of recurrence (1). Gemcitabine (GEM)-based chemotherapy is the established core of multimodal therapy for pancreatic cancer (5). However, it is rarely curative and only modestly effective against tumor recurrence. We need to focus on the recurrence mechanism which is related to biological cancer cell features, such as high invasiveness and migration and find a novel biomarker which could predict the prognosis in clinical situation.

Pancreatic cancer originates from pancreatic ductal epithelium and acquires the characteristics of mesenchymal cells of invading and the metastasizing process. It suggests the existence of epithelial-mesenchymal transition (EMT) during cancer progression (6). The phenomenon of EMT was originally identified during an early embryonic development program in which cells migrate to and colonize different embryonic territories (7). Subsequently, EMT was reported as a similar situation in which cancer cells progress and invade into lymphatic tubes and microvessels (8) and it was shown to be a crucial event during cancer progression and metastasis $(9,10)$. Tumor cancer cells that undergo EMT show reduced intercellular adhesion with decreased expression of E-cadherin and translocation of $\beta$-catenin from cell membrane to the nuclei, and obtain fibroblast-like properties with increased mesenchymal marker expression, such as fibronectin, vimentin and $\mathrm{N}$-cadherin. EMT leads to increased migratory and invasive properties and facilitates metastasis (8-12). Especially in pancreatic cancer, it 


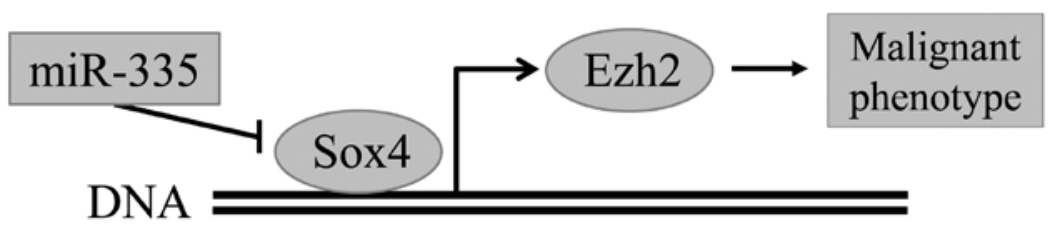

Figure 1. The scheme of Sox4, Ezh2 and miR-335 related to malignant phenotype.

has been reported that pancreatic cancer cells undergo EMT at an early stage, such as PanIN (pancreatic intraepithelial neoplasia) stage which is the precursor cancer lesion (13). It was demonstrated that cancer cells had already spread into the liver as a micrometastasis at a PanIN stage in vivo analysis. The high levels of invasive and migratory abilities were proven in pancreatic cancer (13).

EMT requires the expression of various kinds of regulators such as members of the Zeb, Snail and Twist transcription factor families (14-16). Moreover, EMT phenomenon is plastic and the totally reverse phenomenon to EMT, namely MET (mesenchymal-epithelial transition), is essential for regrowing at metastatic sites (17). It suggests that EMT programs are regulated by some epigenome. Recently a novel pathway that elucidates EMT mechanism induced by transforming growth factor- $\beta$ (TGF- $\beta$ ) was reported (18). The authors demonstrated that TGF- $\beta$ stimulated Sox 4 expression, which induced the histone methyltransferase, Ezh2 and reprogramed the epigenome to induce EMT programs. Sox4 regulated existing EMT inducers such as Zeb, Snail and Twist, acting upstream as a master regulator. In addition, other reports have shown that restoration of miR-335 expression interfered with Sox4 expression, suppressing lung and bone metastasis (19) (Fig. 1).

To date, however, no study has clarified the importance of this Sox4/Ezh2 axis in clinical samples of pancreatic cancer. The expression of Sox 4 and Ezh2 could predict the possibility of cancer metastasis and recurrence. Therefore, we evaluated the prognostic significance of Sox4/Ezh2 axis and expression of miR-335 regarding the clinical outcomes of 36 patients with pancreatic cancer in the present study.

\section{Materials and methods}

Study on primary tumor samples. Between March 2007 and October 2012, 92 patients underwent surgery for pancreatic cancer at Osaka University Hospital, Osaka, Japan. Among the patients, 36 consecutive patients who underwent curative resection (R0) with histologically clear margins with no preoperative therapy were enrolled in the present study. The patients were staged before and after surgery according to the criteria of the International Union Against Cancer (UICC). The median follow-up period was 26.4 months (range, 3.8-79.7 months), the 5-year survival rate was $29.0 \%$ and recurrence of the disease was observed in 19 patients. GEM was administered in 21 patients as an adjuvant chemotherapy (1000 mg/m², 3 times/month for 6 months). No radiation therapy was performed during the follow-up period. Table I summarizes the characteristics of the 36 patients. The use of resected samples was approved by the
Table I. Clinicopathological characteristics of the 36 patients.

\begin{tabular}{lc}
\hline Characteristics & Data \\
\hline Age (years) & $68.5 \pm 9.4$ \\
Gender (Male/female) & $21 / 15$ \\
Location (Ph/Pb/Pt) & $10 / 21 / 5$ \\
Lymphatic invasion (+/-) & $26 / 10$ \\
Venous invasion (+/-) & $15 / 21$ \\
Intrapancreatic perineural invasion (+/-) & $29 / 7$ \\
Maximal diameter (mm) & $25.9 \pm 14.6$ \\
Histology (well/mod/por) & $2 / 30 / 4$ \\
pT (T1/T2/T3/T4) & $4 / 4 / 28 / 0$ \\
pN (+/-) & $16 / 20$ \\
pStage (IA/IB/IIA/IIB/III/IV) & $4 / 4 / 12 / 16 / 0 / 0$ \\
Adjuvant therapy (+/-) & $21 / 15$ \\
Recurrence (+/-) & $19 / 17$ \\
\hline
\end{tabular}

$\mathrm{Ph}$, pancreatic head; $\mathrm{Pb}$, pancreatic body; Pt, pancreatic tail; well, well differentiated; mod, moderately differentiated; por, poorly differentiated.

Human Ethics Review Committee of the Graduate School of Medicine, Osaka University (approval number 08226). The meaning of this study was explained to each patient and written informed consent was obtained from all patients included in the study.

Immunohistochemical staining. The immunohistochemical staining for Sox 4 and Ezh2 in 36 pancreatic cancer samples was performed using the method previously described (20). Briefly, formalin-fixed, paraffin-embedded $4 \mu \mathrm{m}$ thick sections were deparaffinized in xylene, then treated with an antigen-retrieval procedure, and incubated in methanol containing $0.3 \%$ hydrogen peroxide to block endogenous peroxidase. After incubation with normal protein block serum, the sections were incubated overnight at $4^{\circ} \mathrm{C}$ with an anti-Sox4 (LifeSpan BioSciences Inc., Seattle, WA, USA) and anti-Ezh2 (Cell Signaling Technology, Danvers, MA, USA) as the primary antibodies. Thereafter, the labeling was revealed with avidin-biotin complex reagents (Vector Laboratories Inc., Burlingame, CA, USA) and diaminobenzidine. All sections were counterstained with hematoxylin. Positivity for Sox 4 staining was defined as detectable nuclear staining of $>50 \%$ of the cancer cells (Fig. 2C). Positivity for Ezh2 staining was defined as detectable nuclear staining or nuclear and cytoplasm staining of the cancer cells (Fig. 3C and D). 

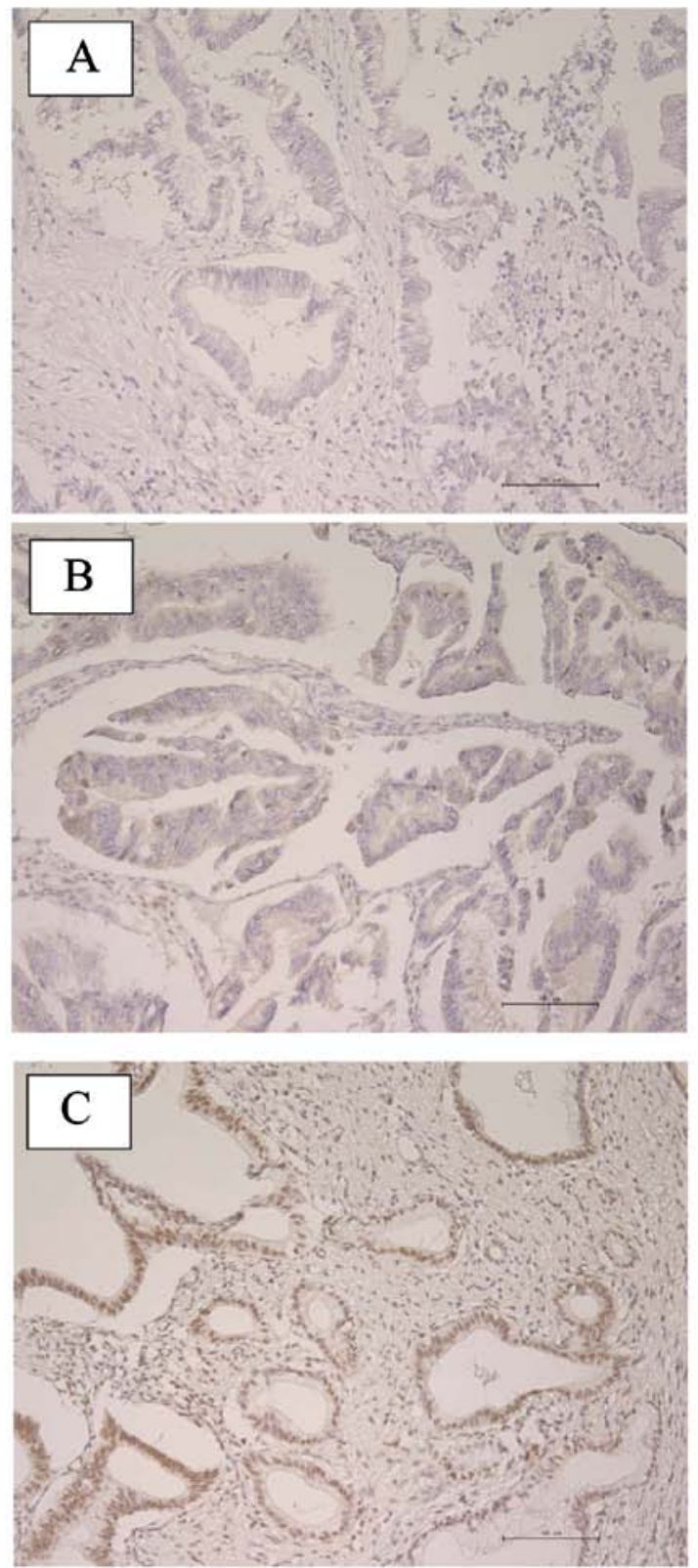

Figure 2. Sox 4 expression in 36 primary pancreatic cancer samples. Immunohistochemical staining of Sox 4 in primary tumor samples. The Sox4-negative cases showed a negative pattern (not stained in nucleus or cytoplasm) (A) or a spotted nuclear pattern (B). (C) Sox4-positive cases showed a defused nuclear pattern. Scale bar, $100 \mu \mathrm{m}$.

Laser captured microdissection (LCM). For LCM, 8- $\mu$ m-thick sections were prepared from the formalin-fixed paraffinembedded (FFPE) samples by utilizing the LCM system (LMD7000; Leica Microsystems GmbH, Wetzlar, Germany) to separate the epithelial and mesenchymal parts from 36 samples, as previously shown (21). Total RNA was extracted using an RNeasy FFPE kit (Qiagen, Tokyo, Japan) with DNase I treatment, according to the manufacturer's instructions. We measured the amount of miR-335 expression in cancer parts with miRNA qRT-PCR. The relative quantification of miRNA expression was calculated using the comparative CT method $\left(2^{-\Delta \mathrm{CT}}\right)(22)$. Data were normalized using RNU48 expression as an endogenous control according to the manufacturer's instruction.
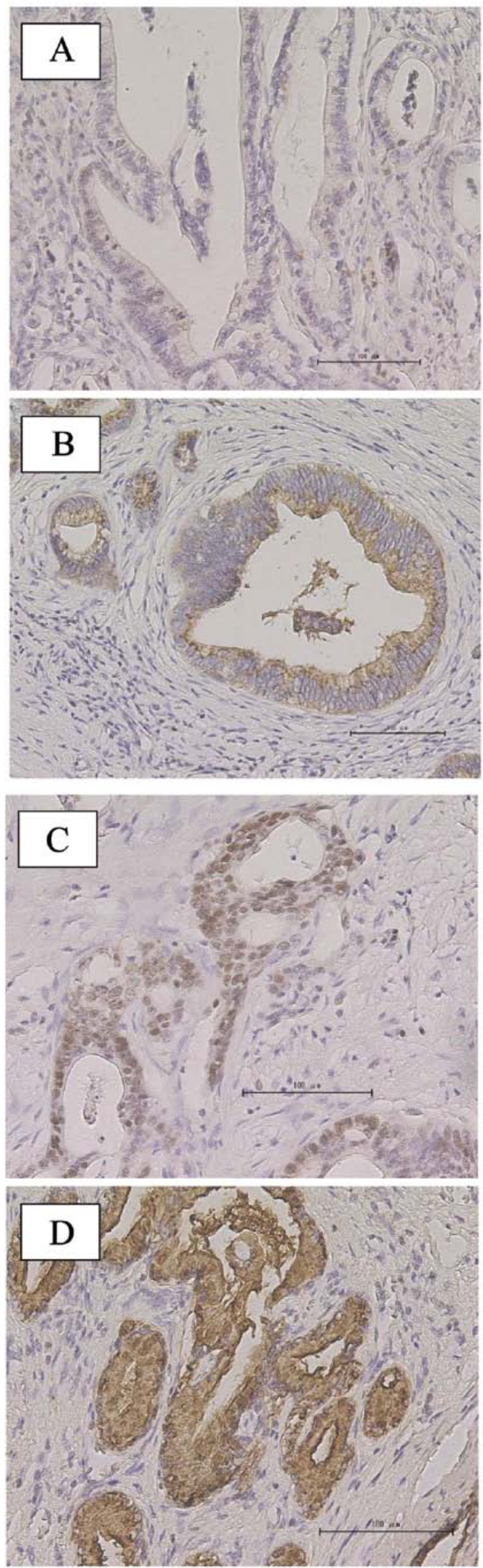

Figure 3. Ezh2 expression in 36 primary pancreatic cancer samples. Immunohistochemical staining of Ezh2 in primary tumor samples. The Ezh2-negative cases showed a negative pattern (not stained in nucleus or cytoplasm) (A) or a cytoplasm pattern (B). Ezh2-positive cases showed a (C) defused nuclear pattern or (D) a nucleus and cytoplasm pattern. Scale bar, $100 \mu \mathrm{m}$ 
A

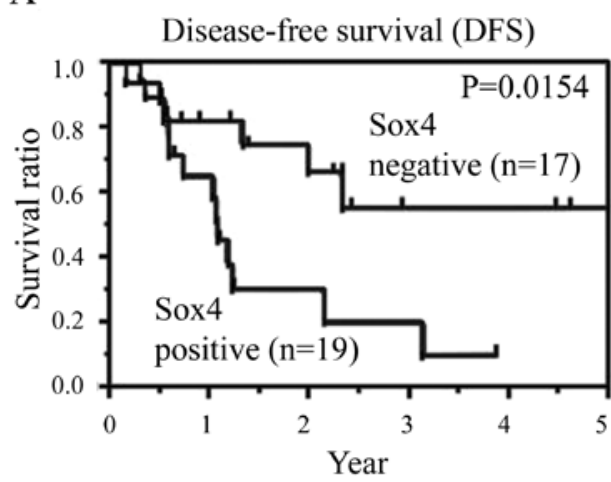

B

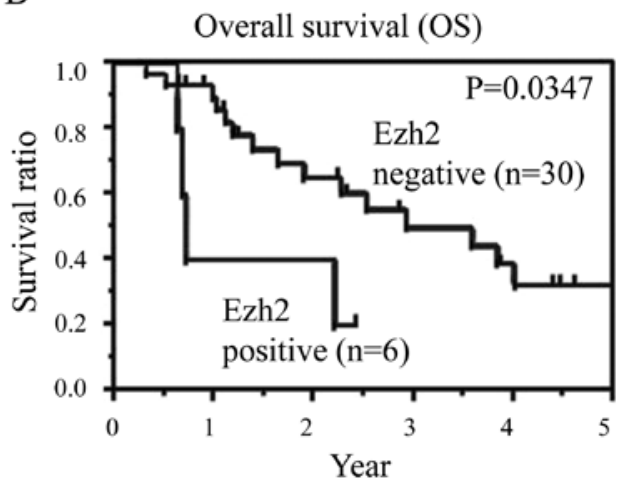

Figure 4. Survival ratio of 36 patients who have underwent complete surgical resection for pancreatic cancer with clear histological margins. (A) Disease-free survival ratio, according to Sox4 expression and (B) overall survival ratio, according to Ezh2 expression.

Statistical analysis. Data were expressed as means \pm standard deviation. The clinicopathological parameters were compared using the Fisher's exact test, and the continuous variables were compared using the Mann-Whitney U test. The survival curves were plotted using the Kaplan-Meier method, and the differences between survival curves were compared using the log-rank test. A P-value of $<0.05$ denoted the presence of a statistically significant difference. Statistical analysis was performed using JMP software version 10.0.2 (SAS Institute Inc., Cary, NC, USA).

\section{Results}

The characteristics of the 36 patients enrolled in the present study. Of the 36 patients in the study, 21 (58.3\%) were males and the mean age of the total patients was $68.5 \pm 9.4$ years (range, 47-83). In $10(27.8 \%)$ patients, tumors were localized in the head and the mean maximal diameter of the tumor was $25.9 \pm 14.6 \mathrm{~mm}$. With the histological type of tumor, most of the patients were moderately differentiated type. Other clinical and histopathological information is listed in Table I.

The clinical significance of Sox4 expression in the tissue samples. The immunohistochemical staining for Sox 4 was performed in the 36 selected samples. In the cancer sections, the functional Sox 4 protein appears to localize in the nucleus. We defined as Sox4-positive cases those in which the cells showed diffused nuclear pattern ( $>50 \%$ of cancer cells) (Fig. 2C), and as Sox4-negative cases those in which the cells showed spotted nuclear pattern $(<50 \%$ of cancer cells) (Fig. $2 \mathrm{~B})$ or a negative pattern (not stained in nucleus or cytoplasm) (Fig. 2A) in the pancreatic cancer lesions. Among the 36 samples examined, $19(52.8 \%)$ samples were positive for Sox 4 , whereas $17(47.2 \%)$ samples were negative.

Subsequently, we studied the relationship between Sox4 expression and clinical outcome. Disease-free survival (DFS) ratio was significantly lower in Sox4-positive group $(\mathrm{P}=0.0154)$ (Fig. 4A) and overall survival (OS) ratio tended to be lower in Sox4-positive group ( $\mathrm{P}=0.0623)$ (data not shown). It might suggest that Sox4 was more deeply associated with invasion and metastasis which were relative to DFS than the refractoriness against several types of therapies such as gemcitabine and S-1.
The clinical significance of Ezh2 expression in clinical tissue samples. The immunohistochemical staining for Ezh2 was also performed in the 36 selected samples. In the cancer sections, we classified the staining pattern into 4 different types (Fig. 3). We defined as Ezh2-positive cases those in which the cells showed diffused nuclear pattern and nuclear and cytoplasm pattern (Fig. 3C and D), and as Ezh2-negative cases those in which the cells showed cytoplasm pattern (Fig. 3B) or a negative pattern (not stained in nucleus or cytoplasm) (Fig. 3A) in the pancreatic cancer lesions. Among the 36 samples examined, only $6(16.7 \%)$ samples were positive for Ezh2, whereas $30(83.3 \%)$ samples were negative. Most of the patients were in the Ezh2-negative group.

Secondly, we assessed the relationship between Ezh2 expression and clinical outcome. OS ratio was significantly lower in Ezh2 positive group ( $\mathrm{P}=0.0347)$ (Fig. 4B), whereas DFS ratio was not $(\mathrm{P}=0.138)$ (data not shown). Therefore, we combined Sox 4 expression with Ezh2 expression and evaluated the relationship between their expression and prognosis. In Sox4- and Ezh2-positive groups, DFS and OS were significantly lower than in the other group (Fig. 5). We were able to identify the patients who had worse prognosis, using these factors.

miR-335 expression of the cancer section in clinical tissue samples. As pancreatic cancer tissues included substantial stromal sections, we performed LCM to collect only cancer sections from tumor tissues with the advice of the pathologists. qRT-PCR was performed to quantify miR-335 expression in the 36 patients (Fig. 6A). The expression level of miR-335 in each sample is shown in Fig. 6B. Of the 36 patients, the mean expression level of miR-335 is 0.28 (/RNU48). We divided the patients into two groups by the mean value of miR-335 expression (high or low). Among the 36 samples examined, 7 $(19.4 \%)$ samples were classified into the high group, whereas $29(80.6 \%)$ were classified into the low group (Fig. 6B).

We evaluated the influence of miR-335 to the prognosis, however, we did not find any significant difference between the expression level of miR-335 and DFS or OS (data not shown).

Sox4 expression and clinicopathological characteristics. We focused on Sox4 expression and evaluated the clinical and histopathological factors between Sox4-positive and -nega- 

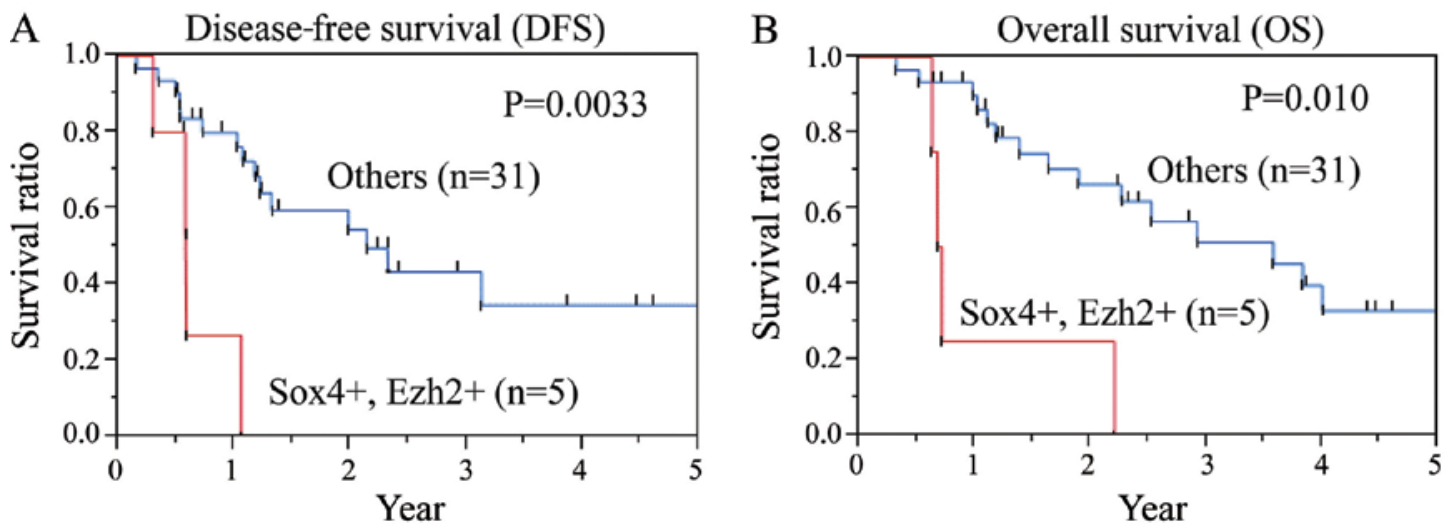

Figure 5. (A) Disease-free survival and (B) overall survival ratios of 36 patients who underwent complete surgical resection for pancreatic cancer with clear histological margins, according to Sox4 and Ezh2 expression.
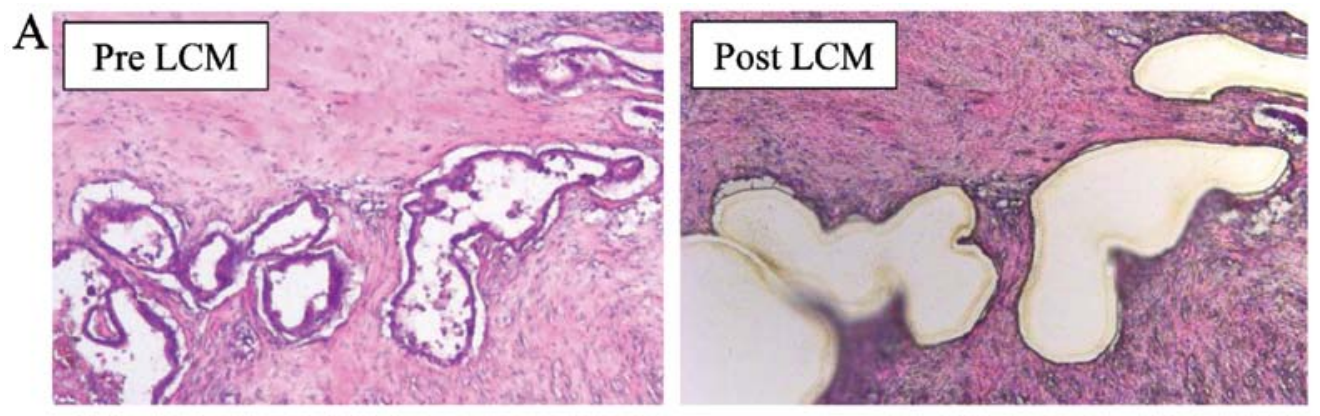

$\mathrm{B}$

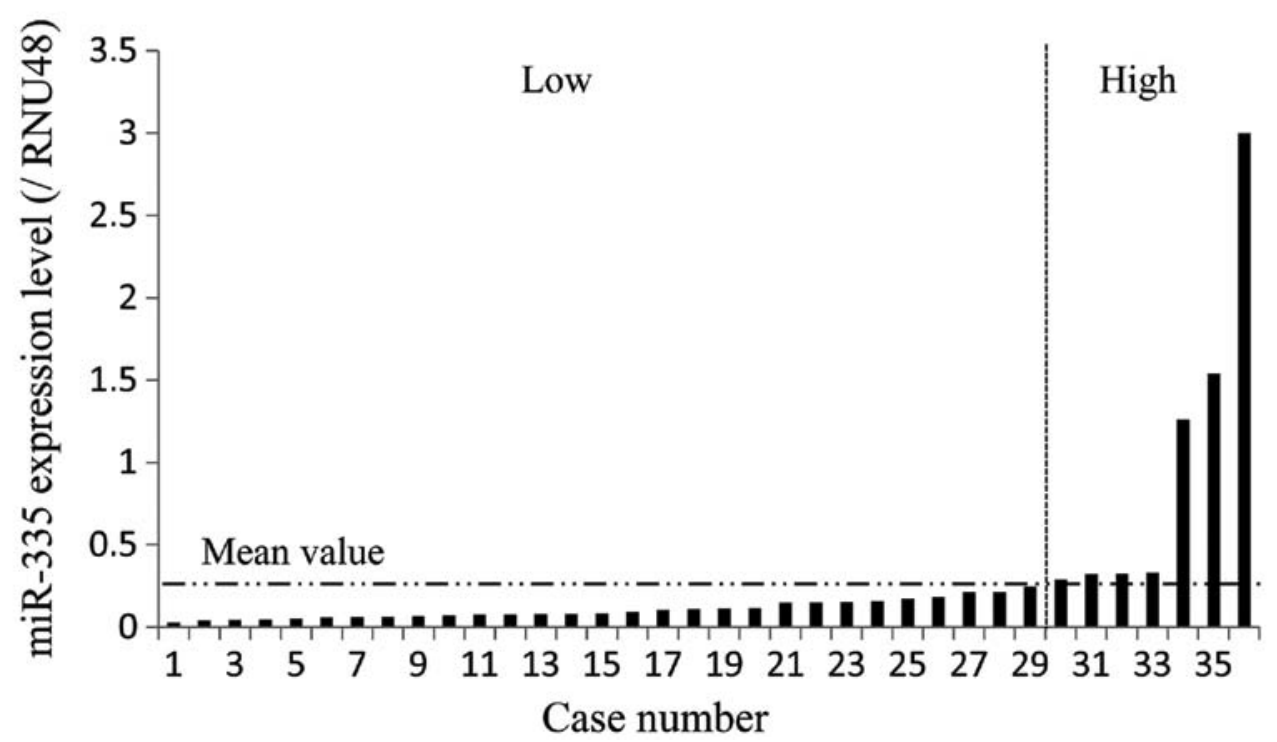

Figure 6. miR-335 expression in clinical pancreatic cancer samples. (A) LCM was performed to collect cancer sections from 36 specimens. The representative image of pre/post (left/right) laser captured microdissection (LCM). (B) Real-time qRT-PCR demonstrated the expression level of miR-335 in each sample. Relative quantification of miRNA expression was calculated by the comparative CT method $\left(2^{-\mathrm{ACT}}\right)$. The mean value of these samples was calculated and divided into two groups with high or low expression of miR-335.

tive groups in order to study the correlation between Sox 4 expression and cancer progression, such as lymphatic invasion, venous invasion, intrapancreatic perineural invasion, and pN (lymph node metastasis) (Table II). The histopathological analysis revealed that these factors were not significantly different between Sox4-positive and -negative groups. The pathological stage was not significantly different either. In brief, Sox 4 expression did not correlate with existing cancer progression factors in pancreatic cancer. However, the expression level of miR-335 which targeted Sox 4 was inversely correlated with Sox 4 expression $(\mathrm{P}=0.0365)$ and 5 out of 6 Ezh2-positive patients (83.3\%) were in Sox4-positive group. It might suggest that miR-335 regulated Sox4 expression and Sox4 promoted Ezh2 expression as a transcription factor. 
Table II. The comparison of clinicopathological factors between the Sox4-positive and -negative groups.

\begin{tabular}{lcc}
\hline & \multicolumn{2}{c}{ Sox4 expression } \\
\cline { 2 - 3 } & Positive (n=19) & Negative (n=17) \\
\hline Age (years) & $66.9 \pm 10.2$ & $70.3 \pm 8.5$ \\
Gender (Male/female) & $12 / 7$ & $9 / 8$ \\
Location (Ph/Pb/Pt) & $10 / 7 / 2$ & $11 / 3 / 3$ \\
Maximal diameter (mm) & $29.6 \pm 17.3$ & $21.8 \pm 9.6$ \\
Histology (well/mod/por) & $0 / 15 / 4$ & $2 / 15 / 0$ \\
Lymphatic invasion (+/-) & $15 / 4$ & $11 / 6$ \\
Venous invasion (+/-) & $9 / 10$ & $6 / 11$ \\
Intrapancreatic perineural invasion (+/-) & $17 / 2$ & $12 / 5$ \\
pT (T1,T2/T3,T4) & $4 / 15$ & $4 / 13$ \\
pN (+/-) & $11 / 8$ & 0.736 \\
pStage (IA, IB/IIA, IIB) & $4 / 15$ & 0.142 \\
Ezh2 expression (+/-) & $5 / 14$ & 0.463 \\
miR-335 expression (High/low) & $1 / 18$ & 0.516 \\
Adjuvant therapy (+/-) & $12 / 7$ & 0.219 \\
Recurrence (+/-) & $13 / 6$ & 0.882 \\
\hline
\end{tabular}

$\mathrm{Ph}$, pancreatic head; $\mathrm{Pb}$, pancreatic body; Pt, pancreatic tail; well, well differentiated; mod, moderately differentiated; por, poorly differentiated

Table III. The predictive markers for disease-free survival in the clinicopathological information.

\begin{tabular}{|c|c|c|c|c|}
\hline & \multicolumn{2}{|c|}{ Univariate analysis } & \multicolumn{2}{|c|}{ Mutivariate analysis } \\
\hline & $\mathrm{HR}(95 \% \mathrm{CI})$ & P-value & $\mathrm{HR}(95 \% \mathrm{CI})$ & P-value \\
\hline Age (years) $(\geq 69 /<69)$ & $2.57(0.97-8.12)$ & 0.0585 & & \\
\hline Gender (Male/female) & $0.55(0.22-1.36)$ & 0.193 & & \\
\hline Maximal diameter $(\mathrm{mm})(\leq 26 />26)$ & $1.11(0.39-2.85)$ & 0.837 & & \\
\hline Histology (well, mod/por) & $0.77(0.21-4.76)$ & 0.746 & & \\
\hline $\mathrm{pT}(\mathrm{T} 1, \mathrm{~T} 2 / \mathrm{T} 3, \mathrm{~T} 4)$ & $0.24(0.054-0.78)$ & 0.0150 & $4.97(0.83-37.00)$ & 0.0794 \\
\hline $\mathrm{pN}(+/-)$ & $5.65(2.04-17.24)$ & 0.0009 & $2.79(0.88-10.47)$ & 0.0813 \\
\hline Lymphatic invasion (+/-) & $2.23(0.80-7.89)$ & 0.132 & & \\
\hline Venous invasion $(+/-)$ & $3.14(1.24-8.33)$ & 0.0160 & $1.00(0.33-3.41)$ & 0.994 \\
\hline Intrapancreatic perineural invasion $(+/-)$ & $2.66(0.76-16.87)$ & 0.140 & & \\
\hline Sox4 expression $(+/-)$ & $3.22(1.24-9.37)$ & 0.0158 & $4.65(1.42-19.42)$ & 0.0096 \\
\hline Ezh2 expression $(+/-)$ & $2.29(0.64-6.52)$ & 0.182 & & \\
\hline miR-335 expression (High/low) & $1.80(0.58-4.79)$ & 0.287 & & \\
\hline
\end{tabular}

Well, well differentiated; mod, moderately differentiated; por, poorly differentiated. HR, hazard ratio; CI, confidence interval.

Relationship between Sox4 expression and prognosis. We assessed the predictive markers for DFS in the clinicopathological information. Upon univariate analysis, pT (tumor invasion depth), $\mathrm{pN}$, venous invasion, and Sox4 expression were significantly associated with DFS while other prognostic markers were not (Table III). Multivariate analysis identified Sox4 expression as a significant and independent prognostic factor $(\mathrm{P}=0.0096)$.

\section{Discussion}

In the present study we assessed the expressions of Sox4, Ezh2 and miR-335 in the identical clinical specimens and evaluated the relationship between the markers and several histopathological factors related to clinical outcome. Among them, Sox 4 reflected the cancer progression most sensitively. Probably it is related to the biological features of Sox 4 molecule and 
increased Sox4 expression might be the trigger of the EMT phenomenon.

Sox4 is included in the SRY-related HMG-box (Sox) family of transcriptional factors, which have a crucial role in embryonic development and cell fate during organogenesis including the pancreas $(23,24)$. Sox4 expression is also increased in many types of cancer, and contributes to cell survival (25), cellular transformation (26) and cancer metastasis (27). In addition, Sox 4 directly regulates important molecules, such as epidermal growth factor receptor (EGFR), Patched-1 (Ptch1), Delta-like 1 (Dll1), tenascin-C, heat shock protein 70 (Hsp70), Foxa1, Dicer and RNA helicase A (28). Moreover, Sox4 regulates Wnt pathway by directly binding to $\beta$-catenin (29). Most recently, Sox4 has been reported to induce EMT in cholangiocarcinoma (30), prostate (31) and breast cancer (32). However, there is no report on Sox 4 and pancreatic cancer prognosis.

In the present study, $52.8 \%$ of the samples expressed Sox4 highly at the nuclei. This finding will not contradict the previous results that pancreatic cancer cells induce EMT at a very early stage. The ratio of the patients who had lymph node metastasis was obviously high in Sox4-positive group (57.9\%) compared with the negative group (29.4\%) (Table II). Moreover, Sox4-positive patients had significantly poor DFS and tended to have worse OS. The results clarify the significance of Sox4 molecule in pancreatic cancer progression. However, few patients expressed Ezh2 highly at the nuclei (16.7\%). It was due to multiple targets of Sox 4 or there might be a time lag during Sox 4 and Ezh2 protein production. Approximately $80 \%$ of Ezh2-positive patients expressed Sox 4 strongly. The data showed the importance of the correlation between Sox 4 and Ezh2 in pancreatic cancer (Fig. 1).

The histone methyltransferase, enhancer of Zeste homolog 2 (Ezh2) is a component of the polycomb repressive complex 2 (PRC2), which epigenetically regulates genes involved in cell fate determination (33). Ezh2 specifically trimethylates nucleosomal histone H3 at lysine 27 (H3K27me3), and inhibits gene expression with an epigenetic modification (34). Ezh2 is expressed highly in a variety of cancers, resulting in malignant transformation and cancer progression (35-37). Furthermore, Ezh2 can induce EMT, and increases the metastatic ability in prostate cancer cells $(36,38)$.

In the present study, Sox 4 and Ezh2 double-positive group had a worse prognosis in DFS and OS ratios; we were able to predict clinical outcomes from the expression levels. In the lethal cancer, pancreatic adenocarcinoma, it could link to the improvement of the clinical outcomes that multimodal therapies were performed on patients predicted to have poor prognosis. On the other hand, miR-335 was not associated with the prognosis, but it related to Sox 4 expression inversely, which might suggest that miR-335 targeted the Sox4 gene.

In conclusion, Sox4/Ezh2 axis is associated with cancer progression and Sox 4 is a novel, independent prognostic factor in pancreatic cancer.

\section{Acknowledgements}

We thank the members of our laboratories for their contribution. The present study was supported in part by a Grant-in-Aid for Scientific Research and a grant from the Platform for Drug Discovery, Informatics, and Structural Life Science, from the Ministry of Education, Culture, Sports, Science and Technology; a Grant-in-Aid from the Third Comprehensive 10-year Strategy for Cancer Control, Ministry of Health, Labor and Welfare; a grant from the Kobayashi Cancer Research Foundation; a grant from the Princess Takamatsu Cancer Research Fund, Japan; and a grant from the National Institute of Biomedical Innovation, Japan.

\section{References}

1. Hoyert DL, Heron MP, Murphy SL and Kung HC: Deaths: Final data for 2003. Natl Vital Stat Rep 54: 1-120, 2006.

2. Hidalgo M: Pancreatic cancer. N Engl J Med 362: 1605-1617, 2010.

3. Shrikhande SV, Kleeff J, Reiser C, Weitz J, Hinz U, Esposito I, Schmidt J, Friess $\mathrm{H}$ and Büchler MW: Pancreatic resection for M1 pancreatic ductal adenocarcinoma. Ann Surg Oncol 14: 118-127, 2007.

4. Bilimoria KY, Bentrem DJ, Ko CY, Stewart AK, Winchester DP and Talamonti MS: National failure to operate on early stage pancreatic cancer. Ann Surg 246: 173-180, 2007.

5. Oettle H, Neuhaus P, Hochhaus A, Hartmann JT, Gellert K, Ridwelski K, Niedergethmann M, Zülke C, Fahlke J, Arning MB, et al: Adjuvant chemotherapy with gemcitabine and long-term outcomes among patients with resected pancreatic cancer: The CONKO-001 randomized trial. JAMA 310: 1473-1481, 2013.

6. Thiery JP, Acloque H, Huang RY and Nieto MA: Epithelialmesenchymal transitions in development and disease. Cell 139: 871-890, 2009.

7. Hay ED: An overview of epithelio-mesenchymal transformation. Acta Anat (Basel) 154: 8-20, 1995.

8. Thiery JP: Epithelial-mesenchymal transitions in development and pathologies. Curr Opin Cell Biol 15: 740-746, 2003.

9. Grünert S, Jechlinger $M$ and Beug $H$ : Diverse cellular and molecular mechanisms contribute to epithelial plasticity and metastasis. Nat Rev Mol Cell Biol 4: 657-665, 2003.

10. Huber MA, Kraut N and Beug H: Molecular requirements for epithelial-mesenchymal transition during tumor progression. Curr Opin Cell Biol 17: 548-558, 2005.

11. Satoh K, Hamada S and Shimosegawa T: Involvement of epithelial to mesenchymal transition in the development of pancreatic ductal adenocarcinoma. J Gastroenterol 50: 140-146, 2015.

12. Thiery JP: Epithelial-mesenchymal transitions in tumour progression. Nat Rev Cancer 2: 442-454, 2002.

13. Rhim AD, Mirek ET, Aiello NM, Maitra A, Bailey JM, McAllister F, Reichert M, Beatty GL, Rustgi AK, Vonderheide RH, et al: EMT and dissemination precede pancreatic tumor formation. Cell 148: 349-361, 2012.

14. Polyak K and Weinberg RA: Transitions between epithelial and mesenchymal states: Acquisition of malignant and stem cell traits. Nat Rev Cancer 9: 265-273, 2009.

15. Cano CE, Motoo Y and Iovanna JL: Epithelial-to-mesenchymal transition in pancreatic adenocarcinoma. Sci World J 10: 19471957, 2010.

16. Browne G, Sayan AE and Tulchinsky E: ZEB proteins link cell motility with cell cycle control and cell survival in cancer. Cell Cycle 9: 886-891, 2010.

17. Gunasinghe NP, Wells A, Thompson EW and Hugo HJ: Mesenchymal-epithelial transition (MET) as a mechanism for metastatic colonisation in breast cancer. Cancer Metastasis Rev 31: 469-478, 2012.

18. Tiwari N, Tiwari VK, Waldmeier L, Balwierz PJ, Arnold P, Pachkov M, Meyer-Schaller N, Schübeler D, van Nimwegen E and Christofori G: Sox 4 is a master regulator of epithelial-mesenchymal transition by controlling Ezh2 expression and epigenetic reprogramming. Cancer Cell 23: 768-783, 2013.

19. Tavazoie SF, Alarcón C, Oskarsson T, Padua D, Wang Q, Bos PD, Gerald WL and Massagué J: Endogenous human microRNAs that suppress breast cancer metastasis. Nature 451: 147-152, 2008.

20. Kondo M, Yamamoto H, Nagano H, Okami J, Ito Y, Shimizu J, Eguchi H, Miyamoto A, Dono K, Umeshita K, et al: Increased expression of COX-2 in nontumor liver tissue is associated with shorter disease-free survival in patients with hepatocellular carcinoma. Clin Cancer Res 5: 4005-4012, 1999. 
21. Nishida N, Nagahara M, Sato T, Mimori K, Sudo T, Tanaka F, Shibata K, Ishii H, Sugihara K, Doki Y, et al: Microarray analysis of colorectal cancer stromal tissue reveals upregulation of two oncogenic miRNA clusters. Clin Cancer Res 18: 3054-3070, 2012.

22. Schmittgen TD, Jiang J, Liu Q and Yang L: A high-throughput method to monitor the expression of microRNA precursors. Nucleic Acids Res 32: e43, 2004

23. Restivo A, Piacentini G, Placidi S, Saffirio C and Marino B Cardiac outflow tract: A review of some embryogenetic aspects of the conotruncal region of the heart. Anat Rec A Discov Mol Cell Evol Biol 288: 936-943, 2006.

24. Lioubinski O, Müller M, Wegner M and Sander M: Expression of Sox transcription factors in the developing mouse pancreas. Dev Dyn 227: 402-408, 2003.

25. Aaboe M, Birkenkamp-Demtroder K, Wiuf C, Sørensen FB, Thykjaer T, Sauter G, Jensen KM, Dyrskjøt L and Ørntoft T: SOX4 expression in bladder carcinoma: Clinical aspects and in vitro functional characterization. Cancer Res 66: 3434-3442, 2006.

26. Liu P, Ramachandran S, Ali Seyed M, Scharer CD, Laycock N Dalton WB, Williams H, Karanam S, Datta MW, Jaye DL, et al: Sex-determining region $\mathrm{Y}$ box 4 is a transforming oncogene in human prostate cancer cells. Cancer Res 66: 4011-4019, 2006.

27. Liao YL, Sun YM, Chau GY, Chau YP, Lai TC, Wang JL, Horng JT, Hsiao M and Tsou AP: Identification of SOX4 target genes using phylogenetic footprinting-based prediction from expression microarrays suggests that overexpression of $\mathrm{SOX} 4$ potentiates metastasis in hepatocellular carcinoma. Oncogene 27: 5578-5589, 2008.

28. Scharer CD, McCabe CD, Ali-Seyed M, Berger MF, Bulyk ML and Moreno CS: Genome-wide promoter analysis of the SOX4 transcriptional network in prostate cancer cells. Cancer Res 69: 709-717, 2009.

29. Sinner D, Kordich JJ, Spence JR, Opoka R, Rankin S, Lin SC, Jonatan D, Zorn AM and Wells JM: Sox 17 and Sox4 differentially regulate beta-catenin/T-cell factor activity and proliferation of colon carcinoma cells. Mol Cell Biol 27: 7802-7815, 2007.
30. Wang W, Zhang J, Zhan X, Lin T, Yang M, Hu J, Han B and Hu S: SOX4 is associated with poor prognosis in cholangiocarcinoma. Biochem Biophys Res Commun 452: 614-621, 2014.

31. Wang L, Li Y, Yang X, Yuan H, Li X, Qi M, Chang YW, Wang C, Fu W, Yang M, et al: ERG-SOX4 interaction promotes epithelialmesenchymal transition in prostate cancer cells. Prostate 74 : 647-658, 2014.

32. Parvani JG and Schiemann WP: Sox4, EMT programs, and the metastatic progression of breast cancers: Mastering the masters of EMT. Breast Cancer Res 15: R72, 2013.

33. Marchesi I, Giordano A and Bagella L: Roles of enhancer of zeste homolog 2: From skeletal muscle differentiation to rhabdomyosarcoma carcinogenesis. Cell Cycle 13: 516-527, 2014.

34. Sparmann A and van Lohuizen M: Polycomb silencers control cell fate, development and cancer. Nat Rev Cancer 6: 846-856, 2006.

35. Varambally S, Cao Q, Mani RS, Shankar S, Wang X, Ateeq B, Laxman B, Cao X, Jing X, Ramnarayanan K, et al: Genomic loss of microRNA-101 leads to overexpression of histone methyltransferase EZH2 in cancer. Science 322: 1695-1699, 2008.

36. Chen H, Tu SW and Hsieh JT: Down-regulation of human DAB2IP gene expression mediated by polycomb Ezh2 complex and histone deacetylase in prostate cancer. J Biol Chem 280: 22437-22444, 2005.

37. Cao Q, Yu J, Dhanasekaran SM, Kim JH, Mani RS, Tomlins SA, Mehra R, Laxman B, Cao X, Yu J, et al: Repression of E-cadherin by the polycomb group protein EZH2 in cancer. Oncogene 27: 7274-7284, 2008

38. Min J, Zaslavsky A, Fedele G, McLaughlin SK, Reczek EE, De Raedt T, Guney I, Strochlic DE, Macconaill LE, Beroukhim R, et al: An oncogene-tumor suppressor cascade drives metastatic prostate cancer by coordinately activating Ras and nuclear factor-kappaB. Nat Med 16: 286-294, 2010 